\title{
Echocardiographic measurements of epicardial adipose tissue and comparative ability to predict adverse cardiovascular outcomes in patients with coronary artery disease
}

\author{
Julieta D. Morales-Portano' ${ }^{1}$ Juan Ángel Peraza-Zaldivar ${ }^{1}$ • Juan A. Suárez-Cuenca ${ }^{2,3,4,7}$ • Rocío Aceves-Millán ${ }^{1}$. \\ Lilia Amezcua-Gómez ${ }^{1}$. Carlos H. Ixcamparij-Rosales ${ }^{1} \cdot$ Rafael Trujillo-Cortés $^{1}$ • Rogelio Robledo-Nolasco ${ }^{1}$. \\ Paul Mondragón-Terán ${ }^{5} \cdot$ Rebeca Pérez-Cabeza de Vaca ${ }^{5} \cdot$ Rolando Hernández-Muñoz $^{6}$. \\ Alberto Melchor-López ${ }^{2,3,4,7} \cdot$ Mani A. Vannan ${ }^{8}$. Alberto Francisco Rubio-Guerra ${ }^{4,7}$
}

Received: 15 September 2017 / Accepted: 20 April 2018 / Published online: 2 May 2018

(c) The Author(s) 2018

\begin{abstract}
The present study aimed to compare echocardiography measurements of epicardial adipose tissue (EAT) thickness and other risk factors regarding their ability to predict adverse cardiovascular outcomes in patients with coronary artery disease (CAD). Outcomes of 107 patients (86 males, 21 females, mean age 63.6 years old) submitted to diagnostic echocardiography and coronary angiography were prospectively analyzed. EAT (measures over the right ventricle, interventricular groove and complete bulk of EAT) and left ventricle ejection fraction (LVEF) were performed by echocardiography. Coronary complexity was evaluated by Syntax score. Primary endpoints were major adverse cardiovascular events (MACE's), composite of cardiovascular death, myocardial infarction, unstable angina, intra-stent re-stenosis and episodes of decompensate heart failure requiring hospital attention during a mean follow up of $15.94 \pm 3.6$ months. Mean EAT thickness was $4.6 \pm 1.9 \mathrm{~mm}$; and correlated with Syntax score and body mass index; negatively correlated with LVEF. Twenty-three cases of MACE's were recorded during follow up, who showed higher EAT. Diagnostic ability of EAT to discriminate MACE's was comparable to LVEF (AUROC > 0.5); but higher than Syntax score. Quartile comparison of EAT revealed that measurement of the complete bulk of EAT provided a better discrimination range for MACE's, and higher, more significant adjusted risk (cutoff $4.6 \mathrm{~mm}$, $\mathrm{RR}=3.91 ; 95 \%$ CI 1.01-15.08; $\mathrm{p}=0.04$ ) than the other risk factors. We concluded that echocardiographic measurement of EAT showed higher predicting ability for MACE's than the other markers tested, in patients with CAD. Whether location for echocardiographic measurement of EAT impacts the diagnostic performance of this method deserves further study.
\end{abstract}

Keywords Echocardiography $\cdot$ Epicardial adipose tissue $\cdot$ Syntax score $\cdot$ Left ventricle ejection fraction $\cdot$ Major adverse cardiovascular events $\cdot$ Coronary artery disease

Juan A. Suárez-Cuenca

suarej05@gmail.com

1 Departments of Echocardiography, Hemodynamics and Cardiology. Centro Médico Nacional "20 de Noviembre", ISSSTE, Mexico City, Mexico

2 Department of Clinical Research, Centro Médico Nacional "20 de Noviembre", ISSSTE, 502, San Lorenzo, Col. Del Valle; Del. Benito Juárez, 03100 Mexico City, Mexico

3 Department of Internal Medicine, H.G.Z. No. 58 and No. 8, Social Security Mexican Institute, Mexico City, Mexico

4 Mexican Group for Basic and Clinical Research in Internal Medicine, Mexico City, Mexico
5 Coordination of Research, Centro Médico Nacional " 20 de Noviembre", ISSSTE, Mexico City, Mexico

6 Departamento de Biología Celular y Desarrollo, Instituto de Fisiología Celular, Universidad Nacional Autónoma de México (UNAM), 04510 Mexico City, CDMX, Mexico

7 Department of Internal Medicine, H.G. Xoco, H.G. Ticomán SSDF, Mexico City, Mexico

8 Cardiovascular Imaging, Piedmont Heart Institute, Piedmont Atlanta Hospital, Atlanta, GA, USA 


\section{Introduction}

Epicardial adipose tissue (EAT), a store of visceral fat situated between the myocardium and pericardium, correlates with the extension and severity of coronary artery disease (CAD) and could be relevant for its local induction and/ or progression $[1,2]$. The pro-inflammatory mRNA profile in EAT is comparable to that in the omental adipose tissue [3], while EAT thickness is associated with pro-atherogenic biomarkers, nitric oxide and malondialdehyde (MDA), as previously reported by our group [4]. Therefore, EAT plays a role as a local inflammatory store in patients with CAD.

There is growing interest in the imaging assessment of EAT. Multislice computed tomography is an accurate method to measure the EAT bulk with high spatial resolution, and the possibility to evaluate in turn coronary atherogenesis and calcification [5, 6]. Echocardiography represents a useful method to measure of EAT, although clinical relevance of measurements at different anatomical locations is not clear. Echocardiography determination of EAT thickness provides advantages like: (1) safe, easily reproducible, and non-invasive method which can be routinely performed [6]; (2) echocardiography measure of EAT independently correlates with CAD $[2,7]$ and with plaque vulnerability $[8$, 9], in the context of acute coronary syndrome and chronic unstable angina. Based on such properties, potential ability of echocardiography measured EAT to predict future atherogenic-related adverse events has been explored [9-11].

Several studies have shown that cardiometabolic risk reflected by elevated EAT parallels the increase in other factors like the left ventricular mass and other components of the metabolic syndrome $[12,13]$. Regarding prediction of major adverse cardiovascular events (MACE's), risk markers like EAT, Syntax score, LVEF and BMI have been characterized, and comparable results have been found by separate studies $[9,10,14-18]$.

However, no study has specifically being designed to compare their predictive ability for MACE's, including echocardiography-determined EAT at different anatomical locations. Therefore, the present study was aimed to compare echocardiography measurements of EAT thickness, Syntax score, LVEF and BMI regarding their ability to predict MACE's in patients with CAD.

\section{Methods}

\section{Study population}

This was an observational, longitudinal, single-center study carried out at the National Medical Center '20 de
Noviembre' ISSSTE, a tertiary referral hospital in Mexico City; following referral by health-care units that serve the general population. We initially included 118 consecutive patients $>18$ years old with angina, in the absence of pericardial effusion, previous ICP, coronary artery bypass grafting or aortic and mitral valve repair or replacement, due to the possibility that EAT had been manipulated during the surgical procedure; who were submitted to diagnostic echocardiography and coronary angiography between September 2013 and February 2016, due to suspected CAD, according to the current guidelines [19]. Eleven patients were excluded due to inadequate acoustic screen during echocardiography, incomplete data and/or were lost at follow-up. Final study population was constituted by 107 patients. The study complied with the ethical guidelines of the 1964 Declaration of Helsinki and its later amendments, and was approved by the local Institutional Committees of Research, Ethics and Biosafety (Protocol ID No. 403.2013). All participants provided written informed consent.

\section{Study protocol}

Coronary angiography, CAD characterization and classification of angina were performed at study enrollment. Echocardiography was performed within the first $48 \mathrm{~h}$ of hospital admission. At the same time the following demographic and anthropometric data were acquired: age, sex, current smoking status, BMI [calculated as weight/ height $\left.{ }^{2}\left(\mathrm{~kg} / \mathrm{m}^{2}\right)\right]$ and obesity (defined as a BMI $\geq 30 \mathrm{~kg} /$ $\mathrm{m}^{2}$ ). High blood pressure was defined as blood pressure $\geq 140 / 90 \mathrm{mmHg}$, when blood pressure was the mean of three readings taken with intervals of $5 \mathrm{~min}$ within each reading, and obtained while the patient was in a seated position, or treatment with antihypertensive agents. Dyslipidemia was defined as one or more of the following conditions: (1) total cholesterol $\geq 200 \mathrm{mg} / \mathrm{dL}$; (2) LDL cholesterol $\geq 100 \mathrm{mg} / \mathrm{dL}$; (3) HDL cholesterol $<40 \mathrm{mg} /$ $\mathrm{dL}$; or (4) triglycerides $\geq 150 \mathrm{mg} / \mathrm{dL}$; or hyperlipidemia treatment. Diabetes mellitus was defined according to the guidelines from the American Diabetes Association with one of the following conditions (repeated for confirmation at a separate date): (1) $\mathrm{Hb} \mathrm{A} 1 \mathrm{C} \geq 6.5 \%$; (2) fasting glucose $\geq 126 \mathrm{mg} / \mathrm{dL}$; or (3) 2 -h plasma glucose $\geq 200 \mathrm{mg} /$ $\mathrm{dL}$ during an oral glucose tolerance test. History of CAD was obtained from the clinical record of each patient. All patients received standard recommendations including medications and life-style changes to limit risk factors for CAD, according to the AHA/ACC guidelines. Patients were periodically followed up at a clinic visit every 3 months or were telephonically contacted in case of being residents of any state out of Mexico City. 


\section{Echocardiographic parameters, LVEF and EAT}

Echocardiography was performed using a Philips iE33 cardiac ultrasound system (Philips Medical Systems, Andover, MA, USA) and a Phillips iE33 S5-1 Transducer (Royal Philips, Amsterdam, The Netherlands). Echocardiographic parameters like E/A, E/Ea and Ventricle Mass $\left(\mathrm{g} / \mathrm{m}^{2}\right)$ were measured according to the guidelines of the American Society of Echocardiography. Left ventricle ejection fraction (LVEF) was measured using a modified Simpson method.

To determine EAT thickness, two-dimensional, M-mode and Doppler transthoracic echocardiography were performed in the left lateral position. EAT thickness was defined as the space between the visceral layer of the pericardium and the outer border of the myocardium, which registered on the standard parasternal longitudinal axis and transverse images. The aortic ring was used as the anatomical reference for the large axis parasternal view, and the papillary muscles were used for the short axis. The images were acquired at the end of systole, over ten cardiac cycles. EAT thickness was evaluated at different locations: EAT was measured on the free wall of the right ventricle from both parasternal long axis view (along the midline and perpendicular to the aortic annulus) and short-axis views at mid ventricle (perpendicular to the interventricular septum at midchordal and tip of the papillary muscle). In all of the cases, reproducibility of the measurement was validated using acceptable intraclass correlation coefficients (agreement) for inter-observer reliability.

\section{Coronary angiography, Syntax score and angina classification}

Coronary angiography and stenting were performed as they were clinically indicated. The procedure was carried out according to standard techniques using radial approach. Evaluable vessels were larger than $1.5 \mathrm{~mm}$ and significant CAD was considered if luminal stenosis was $>50 \%$. Coronary stenting was performed according to standard techniques, using drug-eluting stents in all cases (the drug eluted was everolimus in $50 \%$ of the cases, and zotarolimus in the remaining cases). The mean number of stents applied was one per vessel, while the size and length of the stent was determined by the cardiology expert, being $3 \mathrm{~mm}$ diameter, and $20 \mathrm{~mm}$ length stents the most frequently used. Standard dual antiplatelet therapy previous to PCI consisted of aspirin $150 \mathrm{mg} /$ day and clopidogrel $75 \mathrm{mg} /$ day in patients with chronic stable angina. Patients with acute coronary syndrome received an aspirin loading dose of $300 \mathrm{mg}$ and a clopidogrel loading dose of $600 \mathrm{mg}$ immediately before PCI. After PCI, all patients continued dual antiplatelet therapy, with clopidogrel discontinuation after 1 year. Syntax score was calculated according to classical description [20], using dedicated software (http://www.syntaxscore.com/calculator /start.htm). In all cases, coronary artery luminal narrowing was visually evaluated by two independent researchers, as recommended by the American Heart Association/American College of Cardiology. Angina intensity was evaluated according to the Canadian Cardiovascular Society (CCS) score [21].

\section{Follow up and study endpoints}

Follow-up was conducted every three months after 2 years, either via telephone interview or through written reports from programmed medical evaluations (all subjects agreed to be contacted and to provide information during written consent). If telephone contact was not possible or physician visit were delayed $>2$ months, the endpoints were verified through an authorized person previously designed. Primary endpoints consisted of MACE's, including: (1) cardiovascular death, (2) myocardial infarction, defined as the presence of clinical signs with concomitant increases in CK-MB and troponin higher than 3 times the upper reference values with a previous determination within the reference values; (3) unstable angina, prompting an unscheduled visit to an emergency department within $24 \mathrm{~h}$, (4) intra-stent re-stenosis as demonstrated by coronary angiography, (5) episodes of decompensate heart failure requiring hospital attention.

\section{Statistics}

The study was designed to evaluate the ability of EAT thickness to predict MACE's in patients with CAD, as compared with other markers. For descriptive statistics, continuous variables are presented as mean $\pm \mathrm{SD}$, and categorical variables are presented as $n(\%)$ prevalence. Data distributions for the variables were estimated using Kolmogorov-Smirnov test. Pearson's correlation coefficient was applied to determine the relationship between EAT and variables like age, BMI, LVEF y and Syntax score. To evaluate the ability of EAT to predict MACEs, the mean EAT thickness from cases developing MACEs was compared with mean EAT from those without MACEs, using unpaired Student's $t$ tests. Likewise, diagnostic ability to predict MACEs was estimated using a receiver operating characteristic curve and the area under the ROC curve (AUC). Difference in the range distribution of MACE's according to the location of EAT measure is shown through the comparative number of identifiable MACE's at each EAT quartile, either measured on the ventricle free wall or measured as the complete bulk of EAT. Regression analyses (modeling through the 'enter' method) were performed to determinate the probability of MACE's linked to EAT thickness or the other markers, as well as the weight of variables of potential confusion. The relative risk and $95 \%$ confidence intervals (CIs) are shown. 
All statistical analyses were performed using SPSS software, version 23.0 (SPSS Inc., Chicago, IL, USA) for Windows ${ }^{\circ}$, and $\mathrm{p}$ values $\leq 0.05$ (2-tailed) were considered to be statistically significant.

\section{Results}

The study population was constituted by 107 patients, mean age was 63.6 years old ( 86 males, 21 females) whose demographic and clinical characteristics are summarized in Table 1. High blood pressure and dyslipidemia were significantly prevalent. Half of patients reported previous CAD event and current medical consultation was because of chronic stable angina; most of them at class II from the Canadian Cardiovascular Society functional classification.

Further cardiovascular description included image studies like echocardiography measurements and angiographic CAD characterization, results are summarized in Table 2. Mean EAT thickness was $4.6 \mathrm{~mm}$ and no significant functional abnormalities were observed. Coronary angiography showed damage in more than two coronary arteries in most of cases, being the anterior descendent coronary artery mainly affected and low degree of complexity was most common, as evaluated by Syntax score calculation.

The ability of EAT thickness to reflect cardiovascular risk was initially estimated based on its relation with some

Table 1 Demographic and clinical characteristics $(n=107)$

\begin{tabular}{ll}
\hline Age (years) & $63.6 \pm 9.67$ \\
Male [n (\%)] & $86(80.4)$ \\
BMI & $27.7 \pm 3.77$ \\
Smoke & $52(48.6)$ \\
Co-morbidity & \\
High blood pressure & $77(72.0)$ \\
Dyslipidemia & $65(60.7)$ \\
Type 2 diabetes mellitus & $54(50.5)$ \\
Obesity & $25(23.4)$ \\
History of CAD & $52(48.6)$ \\
Type of CAD & \\
Chronic stable angina & $53(49.5)$ \\
Recent myocardial infarction & $25(23.4)$ \\
Unstable angina & $17(15.9)$ \\
Acute myocardial infarction & $12(11.2)$ \\
CCS functional classification of angina & \\
One & $33(30.8)$ \\
Two & $49(45.8)$ \\
Three & $25(23.4)$ \\
\hline
\end{tabular}

Values are shown as either mean \pm standard deviation, or $\mathrm{n}(\%)$

$B M I$ body mass index, $C A D$ coronary artery disease, CCS Canadian Cardiovascular Society
Table 2 Echocardiographic measures and angiographic CAD characteristics

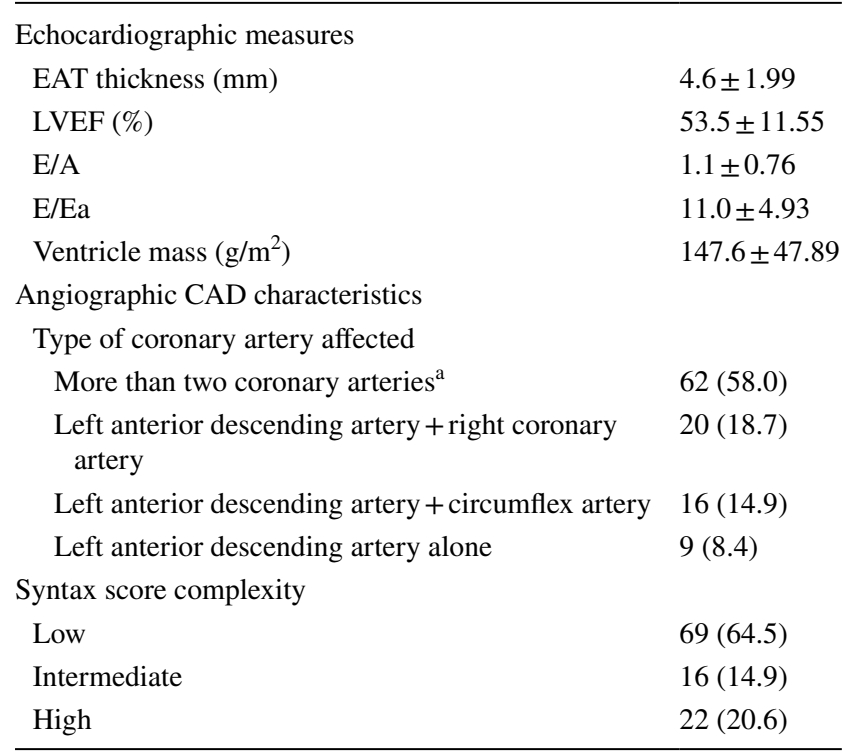

$E A T$ epicardial adipose tissue, $L V E F$ left ventricle ejection fraction, $E / A$ refers to the E/A ratio where $E$ early diastole wave and $A$ enddiastole (atrial contraction) wave, $E / E a$ refers to the index of left atrial pressure

${ }^{a}$ It consistently includes the left anterior descending artery. Values are shown as either mean \pm standard deviation, or $\mathrm{n}(\%)$. Syntax score of complexity of CAD, assessed as low (0-22), intermediate (23-32) and high $(\geq 33)$

markers already studied in previous reports (Fig. 1). We observed that EAT significantly correlated with the complexity of coronary arteries and BMI, while negatively correlated with $\mathrm{LVEF}$.

To evaluate whether EAT thickness might predict development of MACE's, the study population was followed up for a mean time of 16 months after the coronary intervention (15.94 \pm 3.6 months, ranging from 12 up to 24 months), where the number of MACE's were registered. During this time period, 23 MACE's were recorded: two cardiovascular related deaths, 14 hospitalizations due to unstable angina, five cases who required coronary catheterism, two intrastent re-stenosis and five cases with concomitant heart failure requiring hospital attention. According to comparative analysis (Table 3) the group that developed MACE's characterized by significantly higher EAT thickness and lower E/A measures; as well as higher prevalence of diabetes mellitus and chronic stable angina, most of them classified as type II CCS. Likewise, diagnostic performance of EAT to discriminate MACE's was comparable to LVEF, both AUROC's > 0.5; and higher sensitivity than Syntax score; while low specificity values were obtained for all markers (Fig. 2).

In this study, measurements of EAT were performed at several locations in order to test whether it may influence 
Fig. 1 Relation of EAT thickness with cardiovascular risk markers. The graphics show distribution and Pearson's correlation analysis of EAT with age, BMI, LVEF y and Syntax score. Abbreviations EAT epicardial adipose tissue, $B M I$ body mass index, $L V E F$ left ventricle ejection fraction. $p$ value, indicates statistical significance. Artwork was created in GraphPad Prism 5.0
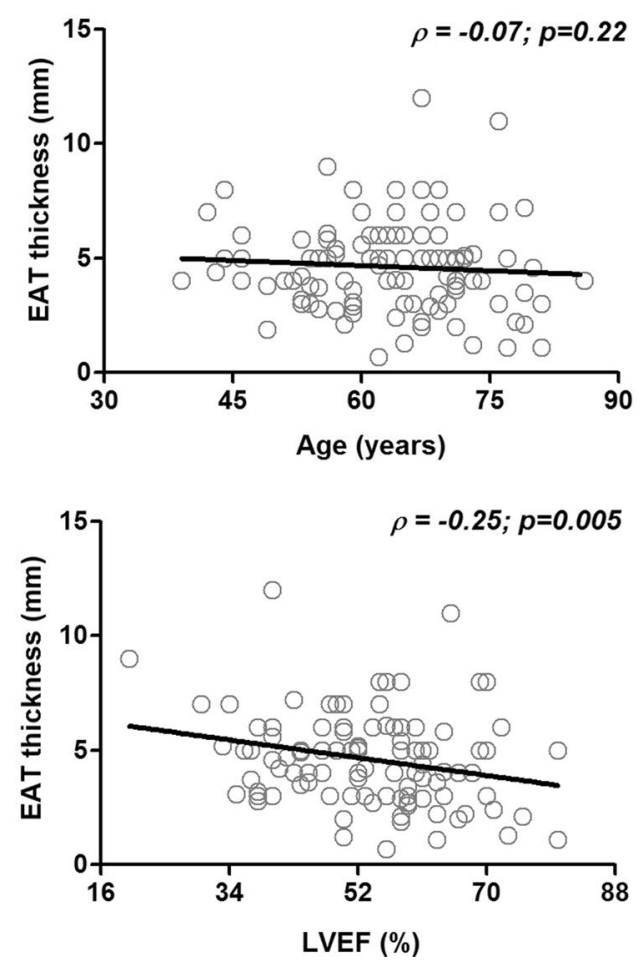
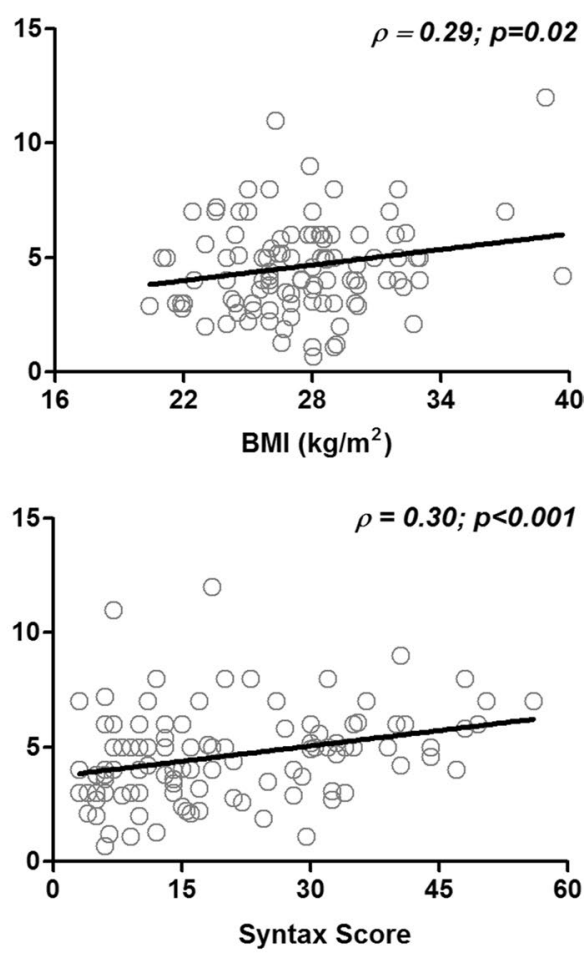

the ability to predict MACEs. Therefore, quartile comparison between values of EAT thickness measures of selective EAT lying over the right ventricle free wall, and measures as complete EAT, was performed (Fig. 3). We observed that measure of complete EAT provides a better discrimination range for MACE's than measure of the EAT lying over the right ventricle free wall.

Finally, adjusted risk for MACE's development attributable to EAT measures at both locations, as well as other potential risk factors, were further compared in a logistic regression analysis (Table 4). The models that included complete EAT measure resulted in a statistically significant increase of almost 4-times $(R R=3.91 ; 95 \%$ CI 1.01-15.08; $\mathrm{p}=0.04)$ the risk of MACE's when complete bulk of $\mathrm{EAT} \geq 4.6 \mathrm{~cm}$.

\section{Discussion}

Our study compared the ability of EAT thickness to predict MACE's in patients with CAD. We found that EAT thickness significantly associated with MACE's development; with a higher risk than the other markers tested.

EAT constitutes a type of VAT with intimate relation with the heart and coronary arteries, which owns unique characteristics regarding adipocyte's hypertrophy, biochemical and metabolic activities [22]. EAT is taught to exert pro-atherogenic effects acting as a source of proinflammatory mediators in a close proximity to coronary arteries and myocardium. Indeed, the relationship between EAT with coronary atherogenesis, plaque volume and stability has been described, as well as its predicting ability for clinical outcome and MACE's [11,23-25]. Consistently, in the present study higher values of EAT were observed in the group that developed MACE's during the follow up period; whereas factors like lower E/A measures, diabetes mellitus and the type of CAD may also be involved. We observed that EAT maintained as independent risk factor for MACE's after weighing potential interactions by logistic regression analysis.

In addition, EAT significantly related with cardiometabolic risk markers like BMI, LVEF and the degree of complexity of coronary arteries, reflected by the Syntax score; being consistent with previous reports also describing MACE's predicting ability of such markers [11, 14, $25,26]$. However, the pathophysiological pathways leading cardiovascular risk underlying to each marker may be different. For example, BMI may be linked to low-grade inflammation and the "obesity paradox" risk phenomenon $[17,18]$; while LVEF may be associated to myocardial injury, ventricular arrhytmia, pump failure with activation of renin-angiotensin-aldosterone and adrenergic systems leading to negative myocardial remodeling and deterioration in contractile function [15] and coronary severity may influence due to the degree of vascular damage, although the relation between coronary stenosis with plaque stability or future MACE's is still debatable [16]. 
Table 3 Comparative characteristics according to MACE's

\begin{tabular}{|c|c|c|}
\hline & Non-MACE's $(\mathrm{n}=84)$ & MACE's $(n=23)$ \\
\hline Age (years) & $63.1 \pm 9.17$ & $65.2 \pm 11.39$ \\
\hline Male $[\mathrm{n}(\%)]$ & $69(82.1)$ & $17(73.9)$ \\
\hline BMI & $27.4 \pm 3.43$ & $28.6 \pm 4.75$ \\
\hline Smoke & $44(52.4)$ & $8(34.8)$ \\
\hline \multicolumn{3}{|l|}{ Co-morbidity } \\
\hline High blood pressure & $62(73.8)$ & $15(65.2)$ \\
\hline Dyslipidemia & $49(58.3)$ & $16(69.6)$ \\
\hline Type 2 diabetes mellitus & $37(44.0)$ & $17(73.9)^{*}$ \\
\hline Obesity & $18(21.4)$ & $7(30.4)$ \\
\hline History of CAD & $38(45.2)$ & $14(60.9)$ \\
\hline \multicolumn{3}{|l|}{ Type of CAD } \\
\hline Chronic stable angina & $37(44.0)$ & $16(69.6)^{*}$ \\
\hline Recent myocardial infarction & $22(26.2)$ & $3(13.0)$ \\
\hline Unstable angina & $15(17.9)$ & $2(8.7)$ \\
\hline Acute myocardial infarction & $10(11.9)$ & $2(8.7)$ \\
\hline \multicolumn{3}{|l|}{ CCS classification of angina } \\
\hline One & $31(36.9)$ & $2(8.7)^{*}$ \\
\hline Two & $29(34.5)$ & $20(87.0)^{*}$ \\
\hline Three & $24(28.6)$ & $1(4.3)^{*}$ \\
\hline \multicolumn{3}{|l|}{ Echocardiographic measures } \\
\hline EAT thickness (mm) & $4.4 \pm 1.81$ & $5.3 \pm 2.43 *$ \\
\hline $\operatorname{LVEF}(\%)$ & $54.2 \pm 11.35$ & $50.3 \pm 12.07$ \\
\hline $\mathrm{E} / \mathrm{A}$ & $1.14 \pm 0.817$ & $0.77 \pm 0.301 *$ \\
\hline $\mathrm{E} / \mathrm{Ea}$ & $10.8 \pm 5.30$ & $12.0 \pm 3.22$ \\
\hline Ventricle mass $\left(\mathrm{g} / \mathrm{m}^{2}\right)$ & $150.5 \pm 48.0$ & $139.8 \pm 48.1$ \\
\hline \multicolumn{3}{|l|}{ Angiographic CAD characteristics } \\
\hline \multicolumn{3}{|l|}{ Type of coronary artery affected } \\
\hline More than two coronary arteries ${ }^{a}$ & $46(54.8)$ & $16(69.6)$ \\
\hline LADA artery + RCA & $19(22.6)$ & $1(4.3)$ \\
\hline $\mathrm{LADA}+\mathrm{CA}$ & $11(13.1)$ & $5(21.8)$ \\
\hline LADA alone & $8(9.5)$ & $1(4.3)$ \\
\hline \multicolumn{3}{|l|}{ Syntax score complexity } \\
\hline Low & $53(63.1)$ & $16(69.6)$ \\
\hline Intermediate & $14(16.7)$ & $2(8.6)$ \\
\hline High & $17(20.2)$ & $5(21.8)$ \\
\hline
\end{tabular}

Values are shown as either mean \pm SD, or $\mathrm{n}(\%)$.Syntax score of complexity of CAD, assessed as low $(0-22)$, intermediate (23-32) and high $(\geq 33)$

$B M I$ body mass index, $C A D$ coronary artery disease, $C C S$ Canadian Cardiovascular Society, EAT epicardial adipose tissue, $L V E F$ left ventricle ejection fraction, $E / A$ refers to the E/A ratio where $E$ early diastole wave and $A$ end-diastole (atrial contraction) wave, $E / E a$ refers to the index of left atrial pressure, $L A D A$ left anterior descending artery, $R C A$ right coronary artery, $C A$ circumflex artery

$* \mathrm{p}<0.05$ two-way test for unequal variances

${ }^{a}$ Consistently includes the LADA
Therefore, we specifically compared EAT's predicting ability of MACE's versus BMI, LVEF and complexity of coronary arteries in a study population with CAD, owning demographic characteristics and cardiovascular risk similar to previous reports [23]. In comparison with the other markers, only EAT thickness resulted significantly higher in patients who developed MACE's; with an estimated risk for
MACE's of almost four. Consistently, higher baseline values of EAT thickness have been predictive of MACE's, and independent association with similar risk values have been obtained in several studies [10, 11, 14, 23, 25]. Moreover, we found that EAT provided acceptable diagnostic performance for MACE's, which was similar to LVEF and with higher sensitivity than Syntax score and BMI. The AUC in 


\section{EAT}

\section{thickness}
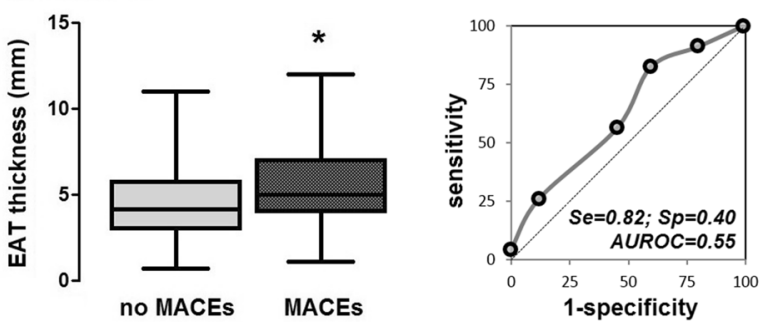

\section{LVEF}
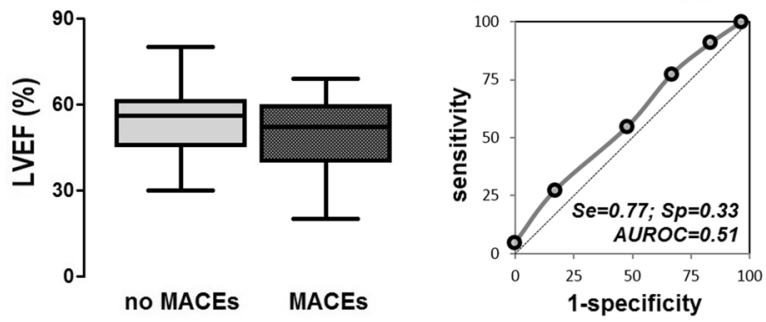

Fig. 2 Comparative ability of EAT thickness to predict MACE's. The boxplots show the values of EAT thickness, BMI, LVEF and Syntax score, as divided by the development of MACE's. The ROC curve indicates the area under the curve (AUROC's), and values (\%) of sen-

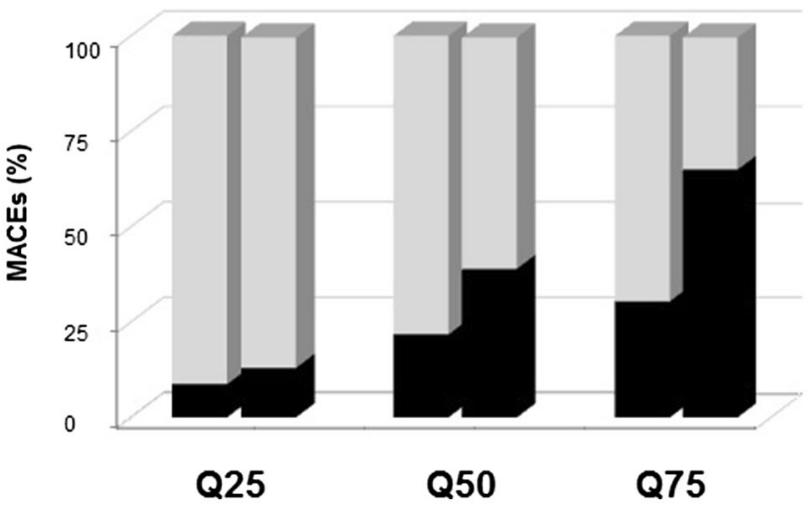

Fig. 3 Range distribution of MACE's according to location of EAT measure. Bars represent the percentage of MACEs identified at every quartile $(\mathrm{Q})$, when EAT thickness was selectively measured over the right ventricle free wall (p25=3; p50=4.6, p75=6) or when measure of complete bulk of EAT was performed (p25 $=3.6 ; \mathrm{p} 50=11$, p75 =19). Abbreviation MACE's major adverse cardiac events. Artwork was created in Excel

our study is lower than other reports [10]. To our knowledge, this is the first study comparing EAT thickness, BMI, LVEF and complexity of coronary arteries regarding their quantification, MACE's association and diagnostic abilities.

In the present study, we performed echocardiographic measure of EAT thickness and used the median value to

\section{BMI}
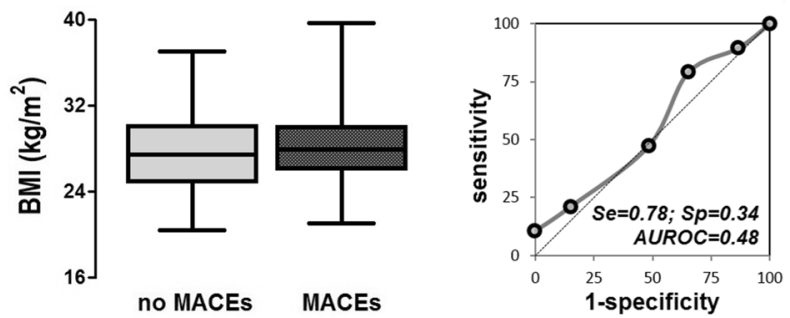

\section{Syntax}

\section{Score}
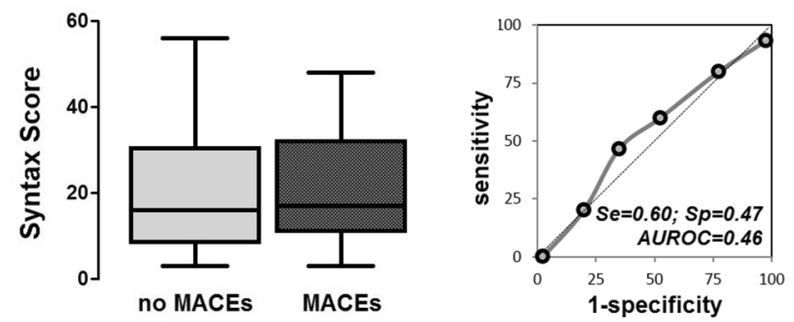

sitivity (Se) and specificity (Sp). $*$ Statistically significant, $\mathrm{p}<0.05$. Abbreviations EAT epicardial adipose tissue, BMI body mass index, LVEF left ventricle ejection fraction. Artwork was created in GraphPad Prism 5.0

estimate the risk for developing MACE's. However, there is a high variability in the way of measure EAT and the value considered of risk for MACE's between studies. Some have used quartiles or 90th percentile of EAT basal measures, or even the EAT remodeling observed between basal measure and a 4-year subsequent measure; obtained through cardiac-CT with measures performed either as parallel, equidistant axial planes or full vertical length images of the heart $[10,25,27]$. Others have used the mean EAT, as echocardiographically determined, or receiver operating characteristics-obtained cutoff value best discriminating MACE's; from measures of the thickest point at the echofree space between the pericardium and the outer wall of the myocardium, performed usually at the free wall of the right ventricle from the parasternal long-axis views [11, 14]. Regardless the measurement method, EAT's predicting ability for MACE's remains highly consistent and risk association values reported are very close to our result.

Moreover, we found that EAT showed a variable discrimination range for MACE's and different prediction ability, related to the location where EAT was measured. This finding has also been previously observed [27]. Although EAT measures on a single slice at specific level of the heart highly correlate with measures of total EAT burden [24], it seems that there is a location-dependent risk, at least observed in the prediction of coronary 
Table 4 Logistic regression analysis of MACE's

\begin{tabular}{llll}
\hline & OR & $95 \%$ CI & $p$ \\
\hline Model 1a. EAT-complete* $^{*}$ & 3.41 & $1.01-10.56$ & $\mathbf{0 . 0 4}$ \\
Model 1b. EAT-complete, adjusted by type of CAD & 3.74 & $1.08-12.88$ & $\mathbf{0 . 0 4}$ \\
Model 1c. EAT-complete, adjusted by T2DM and HBP $^{\text {Model 1d. EAT-right ventricle }}{ }^{\text {a }}$ & 3.91 & $1.01-15.08$ & $\mathbf{0 . 0 4}$ \\
Model 2. Syntax score & 1.90 & $0.72-5.02$ & 0.19 \\
Model 3. BMI & 1.43 & $0.55-3.73$ & 0.46 \\
Model 4. LVEF & 1.13 & $0.42-3.00$ & 0.80 \\
\hline
\end{tabular}

Bold values indicate statistical significance

All models are shown after sex- and age- adjustment

Median cutoff values were: complete EAT measure $4.6 \mathrm{~cm}$, EAT measured over the right ventricle $4.6 \mathrm{~cm}$, Syntax score 16, BMI 28 and LVEF 55\%

$M A C E$ 's major adverse cardiac events, $E A T$ epicardial adipose tissue, $C A D$ coronary artery disease, $T 2 D M$ type 2 diabetes mellitus, $H B P$ high blood pressure, $B M I$ body mass index, $L V E F$ left ventricle ejection fraction

*Measure of the complete EAT

${ }^{\text {a }}$ Measure of the EAT lying over the free wall of the right ventricle atherogenesis. According to studies and meta-analysis, EAT thickness measured at the left $\mathrm{AV}$ groove is associated with obstructive CAD risk [28, 29]; whereas scanty information exists regarding EAT's location-dependent risk for MACE's. Currently, there is no guidelines or consensus regarding its clinical use of EAT measure. Some recommendations are based on its relation with MRI measures or with coronary angiography findings; moreover, echocardiographic cutoff values for increased EAT measures has been suggested [5]. However, echocardiographic method of EAT measure that best reflects its MACE's prediction ability is to be defined.

The strength of our study are both, the performance analysis for MACE's discrimination when echocardiographic EAT measurements are collected at different anatomical references; and the comparison of MACE's prediction risk regarding other markers. However, major limitations of the present study includes: first, potential selection bias because patients were recruited from those referred for echocardiographic examination; second, no survival analysis at different time points for MACE's was performed; and third, the relatively small number of patients and cardiac endpoints.

In conclusion, the ability of EAT thickness in predicting MACE's was better than the other markers tested in a population with CAD. The influence of selective location of EAT measure over MACE's discrimination performance deserves further study due to potential usefulness for cardiovascular outcome prediction.

\section{Compliance with ethical standards}

Conflict of interest The authors declare that they have no conflict of interest.
Ethical approval All procedures performed in studies involving human participants were in accordance with the ethical standards of the institutional and/or national research committee and with the 1964 Helsinki declaration and its later amendments or comparable ethical standards.

Informed consent Informed consent was obtained from all individual participants included in the study.

Open Access This article is distributed under the terms of the Creative Commons Attribution 4.0 International License (http://creativeco mmons.org/licenses/by/4.0/), which permits unrestricted use, distribution, and reproduction in any medium, provided you give appropriate credit to the original author(s) and the source, provide a link to the Creative Commons license, and indicate if changes were made.

\section{References}

1. Taguchi R, Takasu J, Itani Y, Yamamoto R, Yokoyama K, Watanabe $S$ et al (2001) Pericardial fat accumulation in men as a risk factor for coronary artery disease. Atherosclerosis 157:203-209

2. Jeong JW, Jeong MH, Yun KH, Oh SK, Park EM, Kim YK et al (2007) Echocardiographic epicardial fat thickness and coronary artery disease. Circ J 71:536-539

3. Baker AR, Silva NF, Quinn DW, Harte AL, Pagano D, Bonser RS et al (2006) Human epicardial adipose tissue expresses a pathogenic profile of adipocytokines in patients with cardiovascular disease. Cardiovasc Diabetol 5:1-7

4. Peraza-Zaldívar JA, Suárez-Cuenca JA, Aceves-Millán R, Ixcamparij-Rosales C, Amezcua L, Pérez-Cabeza de Vaca R et al (2016) Pro-atherogenic mediators and subclinical atherogenesis are related to epicardial adipose tissue thickness in patients with cardiovascular risk. J Int Med Res 45:1879-1891

5. Bertaso AG, Bertol D, Duncan BB, Foppa M (2013) Epicardial fat: definition, measurements and systematic review of main outcomes. Arq Bras Cardiol 101:e18-e28. https://doi.org/10.5935/ abc. 20130138

6. Salazar J, Luzardo E, Mejías JC, Rojas J, Ferreira A, Rivas-Ríos JR et al (2016) Epicardial fat: physiological, pathological, and 
therapeutic implications. Cardiol Res Pract 2016:1291537. https ://doi.org/10.1155/2016/1291537

7. Yañez-Rivera TG, Baños-Gonzalez MA, Ble-Castillo JL, TorresHernandez ME, Torres-Lopez JE, Borrayo-Sanchez G (2014) Relationship between epicardial adipose tissue, coronary artery disease and adiponectin in a Mexican population. Cardiovasc Ultrasound 12:35. https://doi.org/10.1186/1476-7120-12-35

8. Tanındı A, Kocaman SA, Erkan AF, Uğurlu M, Alhan A, Töre HF (2013) Epicardial adipose tissue thickness is associated with myocardial infarction and impaired coronary perfusion. Anatol J Cardiol 15:224-231. https://doi.org/10.5152/akd.2014.5277

9. Park JS, Choi SY, Zheng M, Yang HM, Lim HS, Choi BJ et al (2013) Epicardial adipose tissue thickness is a predictor for plaque vulnerability in patients with significant coronary artery disease. Atherosclerosis 226:134-139. https://doi.org/10.1016/j.atheroscle rosis.2012.11.001

10. Hajsadeghi F, Nabavi V, Bhandari A, Choi A, Vincent H, Flores F et al (2014) Increased epicardial adipose tissue is associated with coronary artery disease and major adverse cardiovascular events. Atherosclerosis 237:486-489. https://doi.org/10.1016/j.atheroscle rosis.2014.09.037

11. Chu CY, Lee WH, Hsu PC, Lee MK, Lee HH, Chiu CA et al (2016) Association of increased epicardial adipose tissue thickness with adverse cardiovascular outcomes in patients with atrial fibrillation. Medicine 95:e2874. https://doi.org/10.1097/ MD.0000000000002874

12. Iacobellis G, Corradi D, Sharma AM (2005) Epicardial adipose tissue: anatomic, biomolecular and clinical relationships with the heart. Nat Clin Pract Cardiovasc Med 2:536-543

13. Iacobellis G, Ribaudo MC, Zappaterreno A, Iannucci CV, Leonetti F (2004) Relation between epicardial adipose tissue and left ventricular mass. Am J Cardiol 94:1084-1087

14. Park JS, Lee YH, Seo KW, Choi BJ, Choi SY, Yoon MH et al (2016) Echocardiographic epicardial fat thickness is a predictor for target vessel revascularization in patients with ST-elevation myocardial infarction. Lipids Health Dis 15:194

15. Im MS, Kim HL, Kim SH, Lim WH, Seo JB, Chung WY Other Korea Acute Myocardial Infarction Registry (KAMIR) and Korea Working Group on Myocardial Infarction (KorMI) Investigators et al. (2016) Different prognostic factors according to left ventricular systolic function in patients with acute myocardial infarction. Int J Cardiol 221:90-96. https://doi.org/10.1016/j.ijcar d.2016.06.100

16. Pan HC, Sheu WH, Lee WJ, Lee WL, Liao YC, Wang KY et al (2015) Coronary severity score and C-reactive protein predict major adverse cardiovascular events in patients with stable coronary artery disease (from the Taichung CAD study). Clin Chim Acta 445:93-100. https://doi.org/10.1016/j.cca.2015.03.029

17. Kunimura A, Ishii $H$, Uetani $T$, Aoki $T$, Harada $K$, Hirayama $K$ et al (2017) Impact of nutritional assessment and body mass index on cardiovascular outcomes in patients with stable coronary artery disease. Int J Cardiol 230:653-658. https://doi.org/10.1016/j.ijcar d.2017.01.008

18. Thornqvist C, Gislason GH, Køber L, Jensen PF, Torp-Pedersen C, Andersson C (2014) Body mass index and risk of perioperative cardiovascular adverse events and mortality in 34,744
Danish patients undergoing hip or knee replacement. Acta Orthop 85:456-462. https://doi.org/10.3109/17453674.2014.934184

19. Montalescot G, Sechtem U, Achenbach S, Andreotti F, Arden C et al (2013) 2013 ESC guidelines on the management of stable coronary artery disease: the Task Force on the management of stable coronary artery disease of the European Society of Cardiology. Eur Heart J 34:2949-3003. https://doi.org/10.1093/eurheartj/ eht296

20. Sianos G, Morel MA, Kappetein AP, Morice MC, Colombo A, Dawkins K et al (2005) The STX score: an angiographic tool grading the complexity of coronary artery disease. EuroIntervention $1: 219-227$

21. Hemingway H, Fitzpatrick NK, Gnani S et al (2004) Prospective validity of measuring angina severity with Canadian Cardiovascular Society class: the ACRE study. Can J Cardiol 20:305-309

22. Sacks HS, Fain JN (2007) Human epicardial adipose tissue: a review. Am Heart J 153:907-917

23. Gitsioudis G, Schmahl C, Missiou A, Voss A, Schüssler A, AbdelAty $\mathrm{H}$ et al (2016) Epicardial adipose tissue is associated with plaque burden and composition and provides incremental value for the prediction of cardiac outcome. A clinical cardiac computed tomography angiography study. PLoS ONE 11:e0155120. https:// doi.org/10.1371/journal.pone.0155120

24. Tran T, Small G, Cocker M, Yam Y, Chow BJ (2014) A single slice measure of epicardial adipose tissue can serve as an indirect measure of total epicardial adipose tissue burden and is associated with obstructive coronary artery disease. Eur Heart J Cardiovasc Imaging 15:423-430. https://doi.org/10.1093/ehjci/jet175

25. Nakanishi K, Fukuda S, Tanaka A, Otsuka K, Jissho S, Taguchi $\mathrm{H}$ et al (2014) Persistent epicardial adipose tissue accumulation is associated with coronary plaque vulnerability and future acute coronary syndrome in non-obese subjects with coronary artery disease. Atherosclerosis 237:353-360. https://doi.org/10.1016/j. atherosclerosis.2014.09.015

26. Chen J, Tang B, Lin Y, Ru Y, Wu M, Wang X et al (2016) Validation of the ability of Syntax and clinical Syntax scores to predict adverse cardiovascular events after stent implantation: a systematic review and meta-analysis. Angiology 67:820-828. https://doi. org/10.1177/0003319715618803

27. Shmilovich H, Dey D, Cheng VY, Rajani R, Nakazato R, Otaki $Y$ et al (2011) Threshold for the upper normal limit of indexed epicardial fat volume: derivation in a healthy population and validation in an outcome-based study. Am J Cardiol 108:1680-1685. https://doi.org/10.1016/j.amjcard.2011.07.031

28. Wu FZ, Chou KJ, Huang YL, Wu MT (2014) The relation of location-specific epicardial adipose tissue thickness and obstructive coronary artery disease: systemic review and meta-analysis of observational studies. BMC Cardiovasc Disord 14:62. https:// doi.org/10.1186/1471-2261-14-62

29. Wang TD, Lee WJ, Shih FY, Huang CH, Chen WJ, Lee YT et al (2010) Association of epicardial adipose tissue with coronary atherosclerosis is region-specific and independent of conventional risk factors and intra-abdominal adiposity. Atherosclerosis 213:279-287. https://doi.org/10.1016/j.atherosclerosis .2010 .07 .055 